\title{
An LED-based visible light communication system for multicast
}

\author{
Jong-Sung Lee, Dae-Hee Lee, Sung-Jin Kim, Chang-Heon Oh \\ Department of Electronics and Communication Engineering, \\ Korea University of Technology and Education, 31253, Cheonan, Korea
}

\begin{tabular}{l}
\hline \hline Article Info \\
\hline Article history: \\
Received Sep 4, 2018 \\
Revised Nov 5, 2018 \\
Accepted Nov 19, 2018 \\
\hline Keywords: \\
Bidirectional communication \\
Eavesdropping \\
ID \\
Multicast \\
Visible light communication \\
(VLC) \\
\hline
\end{tabular}

\begin{abstract}
Visible Light Communication is a communication method that transmits data through light by pulsing an LED at high speed, performing broadcast communication for all devices existing in its coverage. Broadcast-based Visible Light Communication is limited to application to Internet of Things services, where various applications exist, although all receivers can communicate within the range of light sources. Therefore, this paper proposes an LED based Visible Light Communication system for multicast. The proposed system performs individual multicast by participating in communication only with receivers configured to use a specified ID value input at the transmitting side during data transmission. Experimental results show that the receiver can receive files individually according to a specified ID value.
\end{abstract}

Copyright $\odot 2019$ Institute of Advanced Engineering and Science. All rights reserved.

\section{Corresponding Author:}

Jong-Sung Lee,

Department of Electronics and Communication Engineering,

Korea University of Technology and Education, 31253, Cheonan, Korea.

Email: choh@koreatech.ac.kr

\section{INTRODUCTION}

As the Internet of Things (IoT) emerges, new application services using sensor devices are being released [1]. Statista, a statistical agency, predicts that 75.4 billion IoT devices will be distributed worldwide by 2025 [2]. A large number of sensor devices will be applied and distributed in the IoT environment, creating data at an explosive rate, and imposing traffic overload for systems with limited performance capabilities [3]. RF-based wireless communication systems, in particular, are insufficient to accommodate the explosive data traffic from IoT devices due to their limited frequency band. By using the unlicensed broadband spectrum, Visible Light Communication (VLC) is attracting attention as a next generation wireless communication technology solution to overcome these problems [4].

VLC is a method of transmitting data through light by pulsing a Light Emitting Diode (LED) at high speed, and it is impossible to use in non-line-of-sight (NLoS) environments, enabling easier security implementations when providing local area service [5]. With VLC, it is possible to transmit massive amounts of data at a high speed by using the unlicensed frequency band of $400 \sim 800 \mathrm{THz}$, and there is no problem caused by frequency allocation. In addition, there is no interference with the existing Industrial Scientific and Medical (ISM) band, so it is not affected by multi-path fading, and can be implemented in places where electromagnetic use is restricted $[6,7]$.

In previous researches, we implemented a VLC module capable of bidirectional transmission and reception through an OTG cable with a smart device, and confirmed that text, image, voice, and video files were transmitted and received through the developed application. However, problems such as instability of communication due to hardware specifications were found in the experimental process [8]. Since then, broadcasting data transmission studies have been conducted to enable data transmission to a number of devices within the coverage of light sources for application of IoT by optical communication [9]. However, problems such as interference of external light sources and instability of communication due to 
hardware specifications were found during the experiment. In addition, broadcast-based visible light communication may be limited in the IoT environment due to the existence of various application examples. Therefore, this paper proposes a separate multicast VLC between devices. The proposed system assigns ID values to all smart devices, interworking with each visible light communication module, and transmits data by inputting the ID value of the receiving side from the transmitting side, and only receives from the smart device having the corresponding ID value.

The composition of this paper is as follows. In Section 2, we describe the related research of visible light communication, and in Section 3, we explain the proposed system. In Section 4, the proposed system is verified through the experimental environment configuration, and the conclusion is made.

\section{RELATED WORKS}

In November 2011, the Institute of Electrical and Electronics Engineers (IEEE) confirmed the VLCPhysical Layer (PHY) as IEEE 802.15.7. IEEE 802.15.7, a standard for VLC, describes a technology for supporting the mitigation of flicker and dimming in a PHY/ Media Access Control (MAC) layer by dividing infrastructure, mobile, and vehicle environments [10]. At the standardization meeting held in Hawaii in November 2014, a PAR(Project Authorization) for the standardization of Optical Wireless Communication (OWC), composed of Light Fidelity (Li-Fi), LED-ID, and Optical Camera Communication (OCC), was adopted. In November 2014, the IEEE 802.15.7r1 OGC TG started full standardization in January 2015 through the final approval of the IEEE 802 EC. The IEEE 802.15.7r OWC TG is standardizing on systems that receive signals and patterns from LED screens, LED displays, digital signage, or LED light sources using smart devices. As standardization related to visible light communication progresses, various studies such as data rate, security, and interference are proceeding [11, 12].

In 2017, Al-Moliki et al. proposed a key extraction protocol in Orthogonal Frequency Division Multiplexing (OFDM) to solve the problem of eavesdropping on VLC links. The proposed method extracts the key from the samples generated by the OFDM method in the PHY/MAC layer [4]. In a 2018 study, Werfli et al. proposed a $4 \times 4$ imaging MIMO(Multiple-Input Multiple-Output) VLC system that uses multiband Carrierless Amplitude and Phase (m-CAP) modulation based on frequency multiplexing for highspeed data transmission using VLC. The experiment demonstrated a transfer rate of up to $249 \mathrm{Mbs}$ for the proposed system [13]. In another 2018 study, Babar et al. proposed a unary-coded dimming scheme and Forward Error Correction (FEC) coding to provide flicker-free illumination in On-Off Keying (OOK) modulated visible light communication systems. It controlled the demisting of LEDs through a unary-coded dimmering scheme and used FFC to detect and correct errors. Experimental results confirmed an SNR gain of up to $6 \mathrm{~dB}$ [14]. Also in 2018, Nlom et al. reported on channel frequency measurement of a hybrid system combining Power Line Communication (PLC) technology and VLC. The measured results provide an overall characterization, model and spectral analysis of the hybrid PLC-VLC channel [15]. In another 2018 study, Eroglu et al. proposed various solutions to group LEDs according to the received signal strength of each LED and assign them to the user to achieve maximum throughput, proportional fairness and quality of service. The proposed method suppresses the interference caused by simultaneous data transmission of the LEDs and improves the overall signal-to-interference and noise ratio by $2 \sim 5 \mathrm{~dB}[16]$.

\section{MATERIALS AND METHODS}

This section explains the multicast, bidirectional transmission and reception module, and the Android application, according to the ID value in the VLC environment.

\subsection{Multicast According to ID Value}

Figure 1 shows the block diagram of a visible light communication system for the multicast. Smart devices perform three functions through the application. First, a fixed ID value is given to the smart device that receives the data. Thus, when inputting and transmitting the ID value and data from the smart device on the transmitting side, data can be received only by the device having the corresponding ID. Second, a file such as text, image, voice, or video is encoded and decoded for data communication and is transmitted to the VLC module. Third, data is transmitted and received between devices through VLC modules and USB OTG cables. The VLC module modulates and demodulates data to transmit and receive visible light signals through the LED and the Photo Diode(PD). When the smart device transmits file data, the VLC module modulates the data into binary data, and the modulated binary data is transmitted through the LED to the receiving device within the range of the light source. Then, the VLC module modulates the data received through the PD into file data and transmits it to the smart device. 


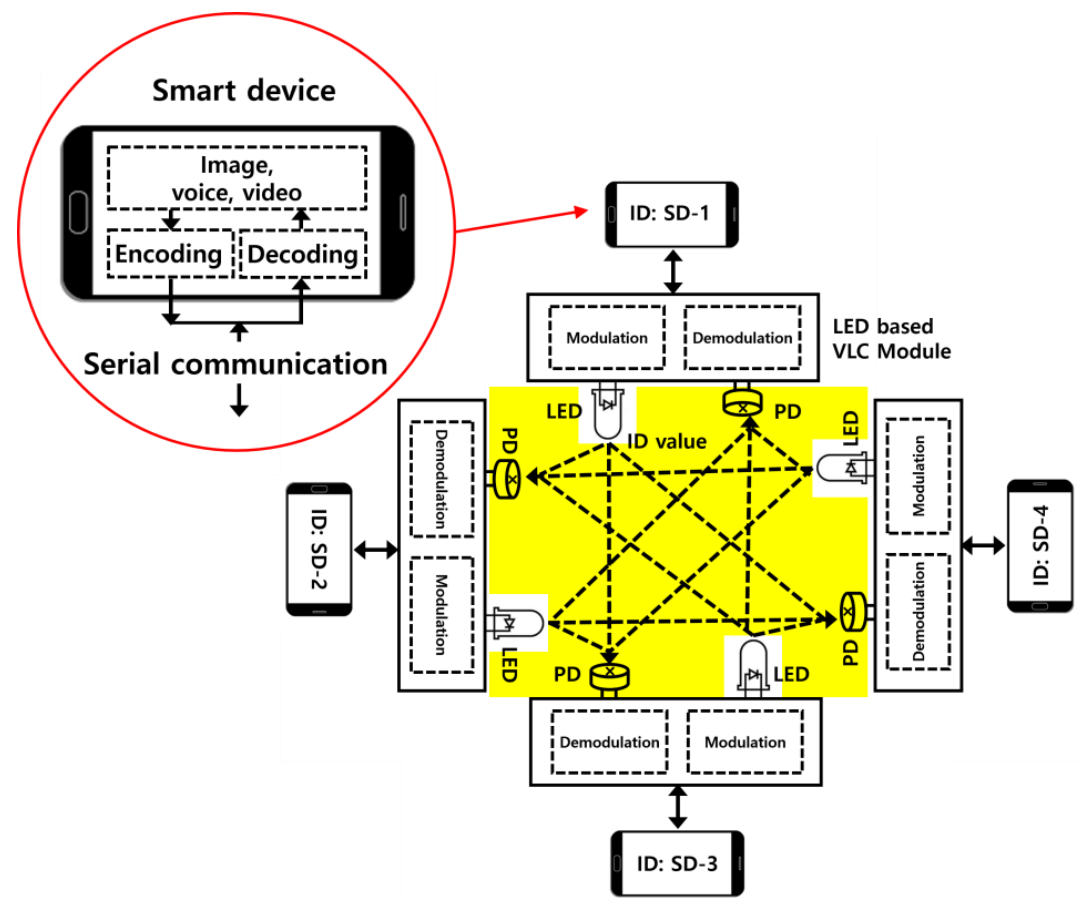

Figure 1. LED-based VLC system for the multicast

Figure 2 shows the data transmission flowchart of the VLC system for the multicast. In the smart device, the user selects the ID value and the file of the device to be transmitted. The selected file is encoded in base64 and converted to text characters with an ID value and transmitted to the VLC module. The VLC module modulates the text value, composed of the ID value and the encoded data, into binary data, and pulses the LED to transmit the visible light signal.

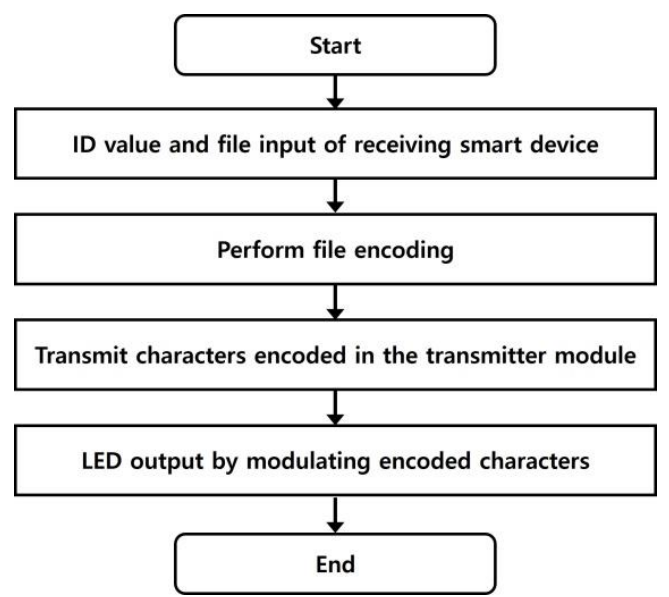

Figure 2. Data transmission flowchart

Figure 3 shows the data reception flowchart of the VLC system for the multicast. The signal received via the PD is demodulated into binary data in the VLC module. The binary data is demodulated as text characters and sent to the smart device through the USB OTG cable. The smart device continuously checks the reception of the data and confirms the data, including the ID value. If the ID value is confirmed, it is compared to the ID value given to the smart device and check whether the ID is verified or not. If the corresponding ID value is matched, then the incoming data is recognized as file data. When all data is received, the incoming data is saved and decoded to create the file. 


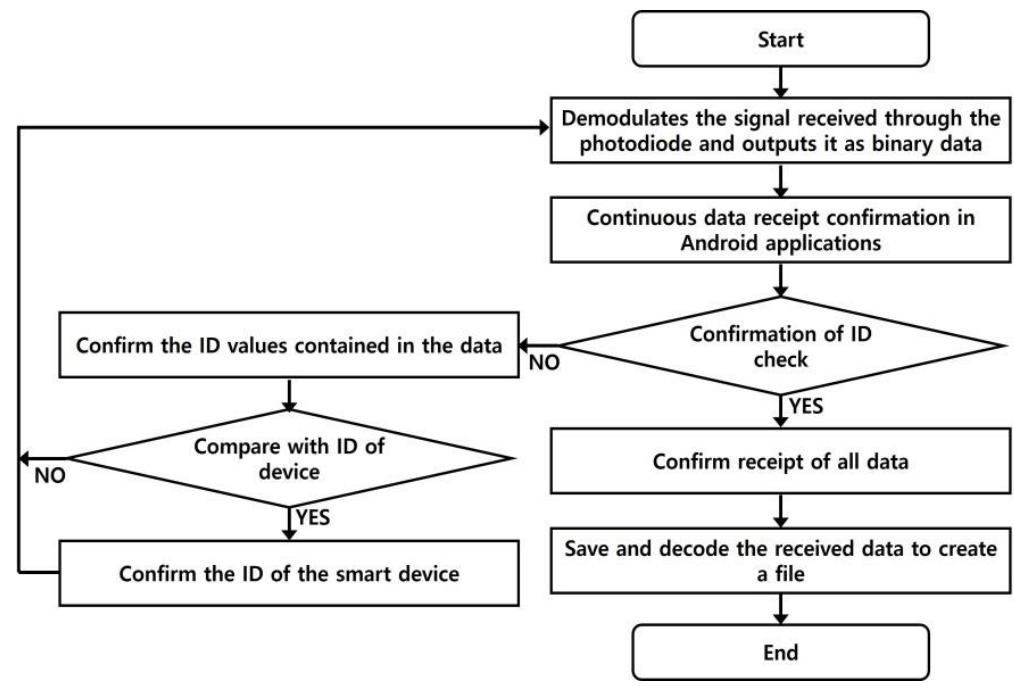

Figure 3. Data reception flowchart

\subsection{Bidirectional Transceiver Module based on VLC}

Figure 4 shows the circuit diagram of the VLC bidirectional transmission and reception module [9]. The VLC-based bidirectional transmission and reception module is based on arduino uno, and performs serial communication using an USB OTG cable for connection with smart devices. The LED converts the data input through serial communication to an analog signal, and switches it through a non-inverting amplifier to a signal with one or more voltage gains without changing the waveform. The visible light signal received by the PD is amplified through a non-release amplifier into a signal with a voltage gain of 1 or more.
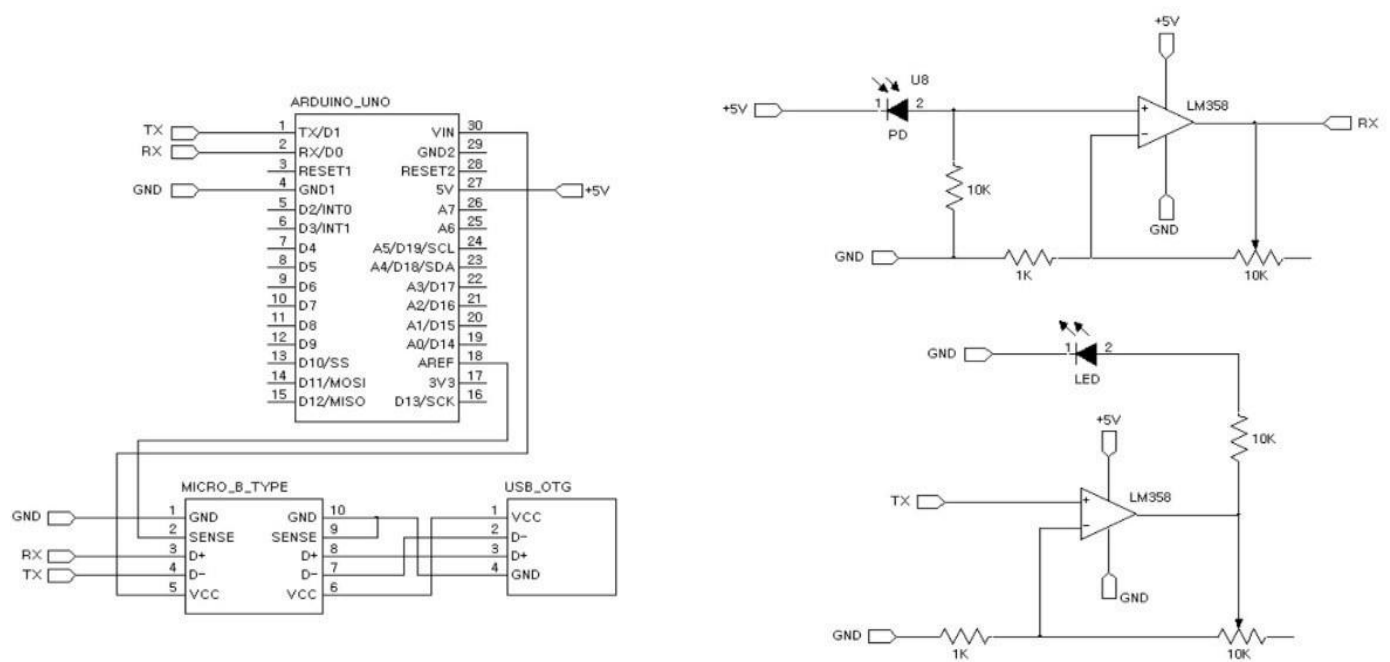

Figure 4. VLC-based bidirectional transceiver module circuit diagram

\subsection{Android Application}

Figure 5 shows a screen for a file transmit application using VLC. When the user transmits a file, the ID value of the smart device to be received and the path and file name of the corresponding file are created in the input box. When the 'SEND' button is clicked, the file is encoded, and the ID value, file name, and encoded data are transmitted to the VLC-based bidirectional transceiver module. When the transmission of the data is completed, the transmitting application outputs a transmission completion message in a text output box below the input box. When the reception of the file is completed, the receiving application outputs a reception complete message, including the ID value in the text view at the bottom, then decodes the received data and stores it as the name of the file in the storage path. 


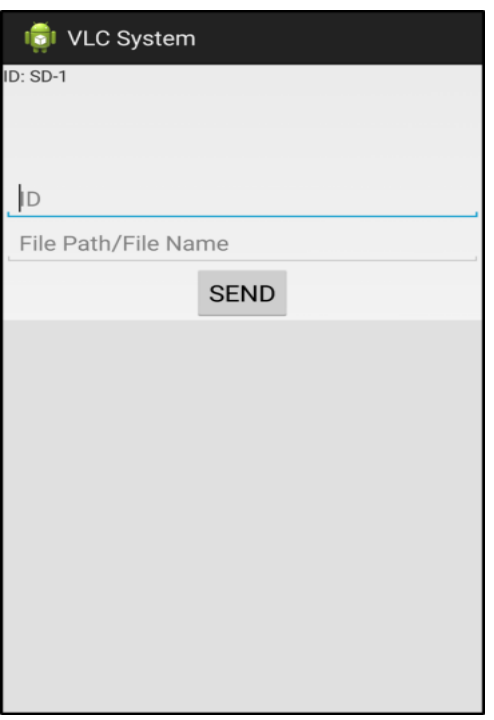

Figure 5. Android application display

\section{EXPERIMENT AND EVALUATION}

\subsection{Experimental Environment}

To verify the performance of the proposed system, the experimental environment is constructed as shown in Figure 6. The smart devices SD-1, 2, 3, and 4 used in the experiment are different Android smart phones. Previous studies have confirmed that data is received from all modules within the range of the LED light source through broadcast-based experiments [9]. This experiment was conducted to transmit different data to the reception device using multicast.

The experimental method transmits files such as image, voice, or video from SD-1 to SD-2, 3, and 4 for performance evaluation on file transmission using the proposed system. Then, the ID values for each device were set equal to the names of SD-1, 2, 3, and 4. The transmitting device's ID value is set to SD-1, and the receiving devices' ID values are set to SD-2, 3, and 4 for the transmission of each image, voice, and video file. Format, file name, and data size for the files to be transmitted in the experiment are presented in Table 1.

Table 1. File Information Used in Experiment

\begin{tabular}{ccc}
\hline File format & File name & Size (byte) \\
\hline png & picture1 & 142 \\
mp3 & voice & 1,985 \\
mp4 & video & 3,960 \\
\hline
\end{tabular}

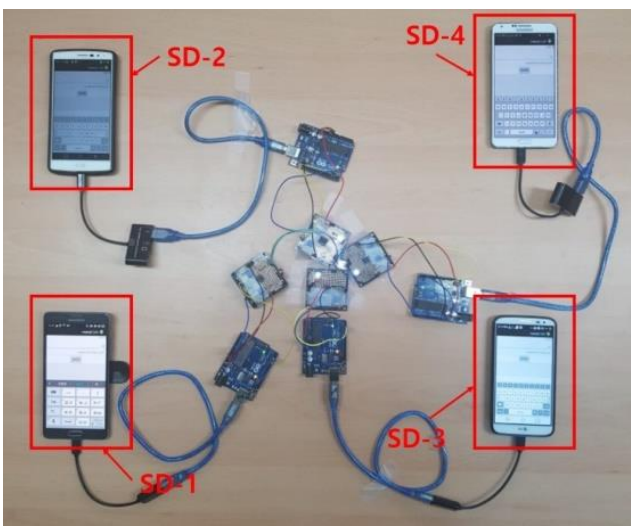

Figure 6. Multicast experimental enviroment 


\subsection{Experiment Result}

Figures 7 (a), (b) and (c) show the output display of SD-2, 3, and 4 after receiving the file data. The reception of ID values transmitted by the transmitter was were confirmed as received through the applications operating in SD-2, 3, and 4. We also checked the reception completion message of the file in the text view of SD-2, 3, and 4 to confirm that the file corresponding to the ID value was received.

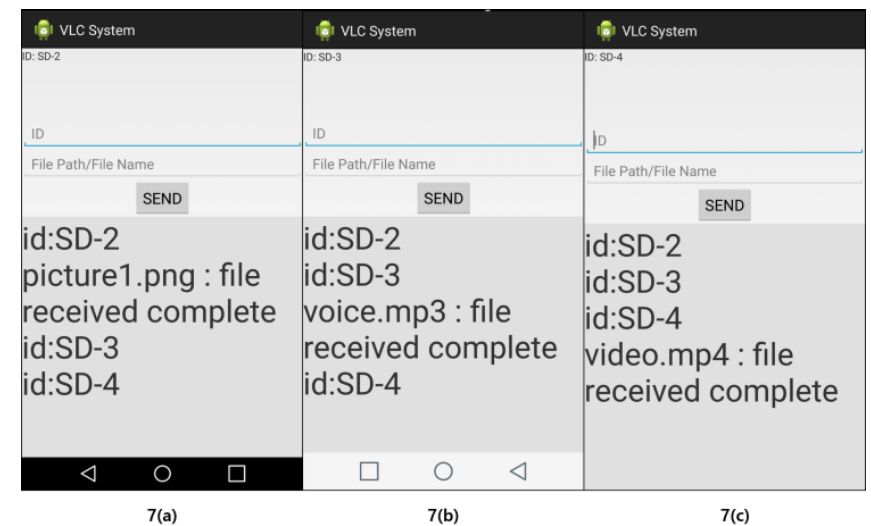

Figure 7. Application display of SD-2,3,4

\section{CONCLUSION}

Due to their limited frequency bandwidth, existing RF communications are insufficient to accommodate the data traffic required by the increased use of IoT devices. In order to supplement such RF communication, visible light communication is attracting attention as one of the next generation wireless communication technologies. However, broadcast-based VLC results in the process of receiving and processing unnecessary data since all receivers within the coverage are able to communicate. This causes unnecessary storage and memory usage in each device, which can be an obstacle to seamless service provision in the IoT environment. Therefore, in this paper, we proposed a visible light communication system for multicast in order to apply VLC to various application services. The proposed system assigns an ID value to a smart device that is used in transmission, then classifies and receives the data according to the ID value of the receiver. Through experiment and evaluation, it was confirmed that various files transmitted from the sender can be transmitted according to the ID value. When the proposed system is used, it is expected that VLC can be applied to various IoT environments.

\section{REFERENCES}

[1] Sharma SK, Wang X. Live data analytics with collaborative edge and cloud processing in wireless IoT networks. IEEE Access. 2017; 5: 4621-4635.

[2] Statista, IoT Connected Devices Installed Based Worldwide from 2015 to 2025 [Internet], Available: https://www.statista.com/statistics/471264/iot-number-of-connected-devices-worldwide/

[3] Munoz R, Vilalta R, Yoshikane N, Casellas R, Martínez R, Tsuritani T, Morita I. Integration of IoT, transport SDN, and edge/cloud computing for dynamic distribution of IoT analytics and efficient use of network resources. Journal of Lightwave Technology. 2018; 36(7): 1420-1428.

[4] Al-Moliki YM, Alresheedi MT, Al-Harthi Y. Secret key generation protocol for optical OFDM systems in indoor VLC networks. IEEE Photonics Journal. 2017; 9(2).

[5] Lee SG, Lee DH, Oh CH. Implementation of VLC relay module to improve communication disconnection phenomenon and coverage expansion in non-line-of-sight area. Journal of the Korea Institute of Information and Communication Engineering. 2018; 22(1): 140-146.

[6] Karunatilaka D, Zafer F, Kalavally V, Parthiban R. LED based indoor visible light communication: state of the art. IEEE communications surveys and tutorials. 2015; 17(3): 1649-1678.

[7] Alao OD, Joshua JV, Franklyn AS, Komolafe O. Light Fidelity(Li-Fi): An emerging technology for the future. IOSR Journal of Mobile Computing \& Application. 2016; 3(3): 18-28.

[8] Lee JS, Kim SJ, Oh CH, LED-based visible light communication system to interacting with device. International Conference on Future Information \& Communication Engineering (ICFICE). Pattaya. 2018; 10(1): 158-160.

[9] Lee JS, Oh CH. LED based visible light communication system using broadcast method. Conference on Korea Institute of Information and Communication Engineering. 2018; 22(1): 641-642. 
[10] IEEE Standards Association. 802.15.7. Short-Range Wireless Optical Communication Using Visible Light. New York: NY; 2011.

[11] Kim SW. IEEE 802.15 WPAN standard trends. The Korean Institute of Communication and Information Sciences. 2015; 32(3): 85-93.

[12] Uysal M, Miramirkhani F, Narmanlioglu O, Baykas T, Panayirci E. IEEE 802.15.7r1 reference channel models for visible light communications. IEEE Communications Magazine. 2017; 55(1): 212-217.

[13] Werfli K, Chvojka P, Ghassemlooy Z, Hassan NB, Zvanovec S, Burton A, Haigh PA, Bhatnagar MR. Experimental demonstration of high-speed $4 \times 4$ imaging multi-CAP MIMO visible light communications. Journal of Lightwave Technology. 2018; 36(10): 1944-1951.

[14] Babar Z, Izhar MAM, Nguyen HV, Botsinis P, Alanis D, Chandra D, Ng SX, Maunder RG, Hanzo L. Unary-coded dimming control improves on-off keying visible light communication. IEEE Transactions on Communications. 2018; 66(1): 255-264.

[15] Nlom SM, Ndjiongue AR, Ouahada K. Cascaded PLC-VLC channel: an indoor measurements campaign. IEEE Access. 2018; 6: 25230-25239.

[16] Eroglu YS, Guvenc I, Şahin A, Yapıcı Y, Pala N, Yüksel M. Multi-element VLC networks: LED assignment, power control, and optimum combining. IEEE Journal on Selected Areas in Communications. 2018; 36(1): 121-135. 